\title{
Scaling universality at the dynamic vortex Mott transition
}

\author{
M. Lankhorst, ${ }^{1}$ N. Poccia, ${ }^{2}$ M. P. Stehno, ${ }^{1}$ A. Galda, ${ }^{3,4}$ H. Barman,,${ }^{5}$ F. Coneri,,${ }^{1}$ H. Hilgenkamp, ${ }^{1}$ A. Brinkman, ${ }^{1}$ \\ A. A. Golubov, ${ }^{1,6}$ V. Tripathi, ${ }^{3,5}$ T. I. Baturina, ${ }^{3,7,8,9}$ and V. M. Vinokur ${ }^{3}$ \\ ${ }^{1}$ MESA+ Institute for Nanotechnology, University of Twente, 7500 AE Enschede, The Netherlands \\ ${ }^{2}$ Department of Physics, Harvard University, Cambridge, Massachusetts 02138, USA \\ ${ }^{3}$ Materials Science Division, Argonne National Laboratory, 9700 South Cass Avenue, Argonne, Illinois 60439, USA \\ ${ }^{4}$ The James Franck Institute and Department of Physics, The University of Chicago, Chicago, Illinois 60637, USA \\ ${ }^{5}$ Department of Theoretical Physics, Tata Institute of Fundamental Research, Homi Bhabha Road, Navy Nagar, Mumbai 400005, India \\ ${ }^{6}$ Moscow Institute of Physics and Technology, Institutskii per. 9, Dolgoprudny 141700, Moscow District, Russia \\ ${ }^{7}$ A. V. Rzhanov Institute of Semiconductor Physics SB RAS, 13 Lavrentjev Avenue, Novosibirsk 630090, Russia \\ ${ }^{8}$ Department of Physics, Novosibirsk State University, Pirogova Street 2, Novosibirsk 630090, Russia \\ ${ }^{9}$ Departamento de Física de la Materia Condensada, Instituto de Ciencia de Materiales Nicolás Cabrera and Condensed Matter Physics \\ Center (IFIMAC), Universidad Autónoma de Madrid, 28049 Madrid, Spain
}

(Received 27 July 2017; revised manuscript received 25 November 2017; published 17 January 2018)

\begin{abstract}
The cleanest way to observe a dynamic Mott insulator-to-metal transition (DMT) without the interference from disorder and other effects inherent to electronic and atomic systems, is to employ the vortex Mott states formed by superconducting vortices in a regular array of pinning sites. Here, we report the critical behavior of the vortex system as it crosses the DMT line, driven by either current or temperature. We find universal scaling with respect to both, expressed by the same scaling function and characterized by a single critical exponent coinciding with the exponent for the thermodynamic Mott transition. We develop a theory for the DMT based on the parity reflection-time reversal $(\mathcal{P} T)$ symmetry breaking formalism and find that the nonequilibrium-induced Mott transition has the same critical behavior as the thermal Mott transition. Our findings demonstrate the existence of physical systems in which the effect of a nonequilibrium drive is to generate an effective temperature and hence the transition belonging in the thermal universality class.
\end{abstract}

DOI: 10.1103/PhysRevB.97.020504

A Mott insulator [1-3] arising from the concurrent action of the electron-electron correlations and electron trapping by a periodic atomic potential is an exemplary manifestation of many-body quantum physics [4-8]. A remarkable correspondence between the quantum mechanics in a $\mathcal{D}$-dimensional system and the classical statistical mechanics of a $\mathcal{D}+1$ dimensional system [9] leads to the conjecture about its classical counterpart, a vortex Mott insulator that would form in a type II superconductor if the density of the superconducting vortices matches the density of the pinning sites [10,11]. Experimentally, the vortex Mott insulator was claimed in the studies of the vortex matching effect in Ref. [12], and was conclusively evidenced in Ref. [13] by measurements of the compressibility of the vortex system localized by periodic surface holes. The implications of the existence of the vortex Mott state are far reaching. First and foremost, the Mottness embraces not only the quantum but classical realm, thus offering a perfect laboratory to study quantum many-body physics by exploring classical vortex systems.

An enabling discovery of the current-driven vortex Mott insulator-to-metal transition in a proximity array [14] provided the first tangible example of a dynamic Mott transition having settled the vortex quantum mechanical mapping on a firm experimental basis. That the revealed nonequilibrium critical behavior with respect to the nonequilibrium drive is the same as that of a conventional thermal Mott transition with respect to temperature raises a largely open class of questions. Among these is a central issue in condensed matter physics: the generalization of a thermodynamic phase transition to nonequilibrium conditions. There have been tantalizing reports that in systems where tuning parameters such as temperature, pressure, or magnetic field alter the symmetry, the nonequilibrium drive generates an effective temperature and the corresponding transition appears in the conventional thermal universality class $[15,16]$. The finding of Ref. [14] paves the way for further generalizing this conclusion to a wider nontrivial class of phase transitions.

A hallmark of an electronic Mott insulator-to-metal transition derived from the Hubbard model [17] is the change in the electronic density of states (DOS) from a gapped (insulator) to peaked (metal) shape $[5,18,19]$ near the Fermi level. Hence, the Mott transition can be detected by measuring the tunneling differential conductance as a function of the particle density and observing the change from a sharp dip, which reflects a depletion of the electronic states at the Fermi level, to a peak, which signals that a Mott metal has formed. An array of vortices trapped in a lattice of pinning sites is a perfect realization of the Hubbard model. In a dual vortex system, the quantum particles-vortex correspondence manifests as a mapping of the tunneling differential conductance of particles onto the thermally activated differential resistance $d V / d I$. Accordingly, the dip-to-peak reversal of the latter measured as a function of the magnetic field heralds the vortex Mott transition [14]. 


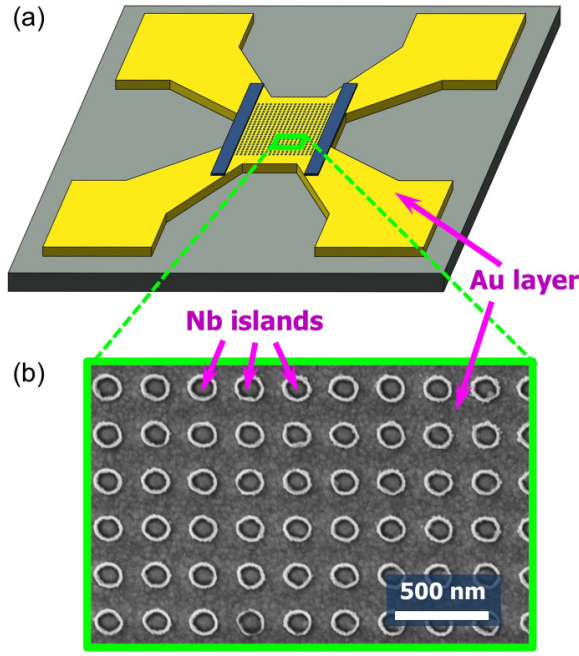

FIG. 1. Experimental realization of charge-vortex duality for a Mott insulator. (a) A sketch of the device. The device consists of a square array of $270 \times 270$ superconducting $\mathrm{Nb}$ islands on a conducting Au layer. On both sides of the array, a Nb bar is placed to ensure the current passes through the array homogeneously. The potential difference between the bars is measured as a function of the external current and an external magnetic field perpendicular to the plane of the array. (b) Scanning electron microscopy image of the sample.

Results. We create an egg-crate periodic pinning potential patterning a square array of $270 \times 270 \mathrm{Nb}$ islands with a lattice constant $a=250 \mathrm{~nm}$ on a 40-nm-thick base layer of $\mathrm{Au}$ on $\mathrm{Si} / \mathrm{SiO}_{2}$, as shown in Fig. 1. The islands are $45 \mathrm{~nm}$ in height and $142 \pm 5 \mathrm{~nm}$ in diameter. Additionally, we placed $\mathrm{Nb}$ bars on either side of the array structure to ensure uniform current injection. The superconducting transition temperature of the array, $T_{c}=2.8 \mathrm{~K}$, is determined as the midpoint of the transition in the temperature-resistance curve. For our square array, the magnetic field at which the number of vortices matches the traps is $B_{0}=\Phi_{0} / a^{2}$, where $\Phi_{0}=\pi \hbar / e$ is the flux quantum. It is convenient to introduce the vortex filling fraction $f=B / B_{0}$, so that $f=1$ corresponds to one vortex per lattice cell. We measure current-voltage characteristics with small steps in magnetic field and temperature and obtain $d V(f) / d I$ curves by numerical differentiation. From these data, the phase boundary was determined by tracking the position of the dipto-peak reversal as a function of current $(I)$ and temperature $(T)$. The details of the measurement technique are given in the Supplemental Material [20]. Figure 2(a) presents the phase diagram of the Mott states in the $T$ - $I$ coordinates summarizing the experimental results of our work. Representative sets of $d V / d I$ curves are shown in Figs. 2(b) and 2(c). These data were taken using a standard lock-in technique near the transition with very small steps of $5 \mu \mathrm{T}$ in magnetic field, $0.5 \mu \mathrm{A}$ in current [Fig. 2(b)], and $5 \mathrm{mK}$ in temperature [Fig. 2(c)]. The isothermal plots of Fig. 2(b) display the expected dip-to-peak reversal upon increasing the current. The separatrix current $I_{0}$ divides between the insulating $I<I_{0}$ and metallic $I>I_{0}$ phases. Note the asymmetry in the $d V / d I$ behaviors at $f<1$ and at $f>1$. The loci of $I_{0}(T)$ yield phase transition lines in Fig. 2(a) for $f<1$ and $f>1$. Fixing the current $I \lesssim I_{0}$ and then varying the temperature yields similar dip-to-peak reversal behavior [see Fig. 2(c)]. Subtracting the separatrices from the $d V / d I$ data yields the fanlike set of curves displayed in Figs. 2(d) and 2(e), indicating a transition from insulating (bent down towards $f=1$ ) to metallic (bent up) behaviors.

Critical scaling. We start our analysis with the following question: Is the observed current-driven dip-to-peak flip indeed a purely dynamic effect, or rather a mere result of the heating due to current-induced vortex motion? To answer it, let us consider the quantity $\mathcal{N}(T, B)=d V / d I-V / I$ that measures the degree of nonlinearity. Figures 3(a) and 3(b) show the color plots of $\mathcal{N}(T, B)$ in coordinates $f-I$ and $f-T$, respectively. The bright red regions ("red flames") indicate domains of strong nonlinearity that arise around $f=1$. Apart from the critical region near the transition, the plots are predominantly blue in color. This shows that the response of the system is almost linear, $d V / d I \approx V / I$. Since dissipation is proportional to $I \cdot V$ and the experiment is carried out at constant $I$, the dissipation is higher where $R$ is larger. Within the experimental range of currents and temperatures across the transition, the resistance grows linearly $R \propto|b| \equiv|1-f|$ upon deviation from $f=1$. This reflects the linear increase of the density of vortex "holes" or the excess vortices that mediate the motion of the vortex system upon deviation from $f=1$ [see Figs. S2(c) and S2(d) in SM], and implies that the mobility of vortices remains nearly unchanged in our experiment. Therefore, had the nonlinearity originated from heating, it could have only increased with increasing $|b|$. The observed effect is the opposite: The nonlinearity associated with a dip-to-peak reversal exists only in the nearest vicinity of $f=1$. Hence the heating from vortex motion is negligible and cannot cause the observed dip-to-peak flip in the differential resistance.

It is worth especially emphasizing that the observed transition identified by nonlinear $I-V$ behavior is not vortex depinning, i.e., eliminating the barriers for vortex motion either by applied current or by thermal fluctuations. This is evidenced by the fact that the measuring currents are considerably lower than the depinning Josephson critical current characterizing the activation barriers [14] and by the above observation that nonlinearity concentrates at integer $f$ where pinning is maximal.

A detailed examination of hundreds of recorded $d V / d I$ curves versus temperature and magnetic field uncovers a striking and far-reaching affinity between current and temperature manifestations in the DMT critical behavior. The scaling analysis of the DMT using the representative set of $d V / d I$ curves from Figs. 2(b) and 2(c) is shown in Fig. 4. The benchmarks of the Mott transition are the scaling relations governing the behavior of $d V / d I$ in the critical region [4-7,14],

$$
\begin{gathered}
\frac{d V}{d I}(b, I, T)-\left.\frac{d V}{d I}(b, I, T)\right|_{I=I_{0}} \propto \mathcal{F}_{\mathrm{I}}\left(\frac{\left|I-I_{0}\right|}{b^{\epsilon_{\mathrm{I}}}}\right), \\
\frac{d V}{d I}(b, I, T)-\left.\frac{d V}{d I}(b, I, T)\right|_{T=T_{0}} \propto \mathcal{F}_{\mathrm{T}}\left(\frac{\left|T-T_{0}\right|}{b^{\epsilon_{\mathrm{T}}}}\right),
\end{gathered}
$$

where $\epsilon_{\mathrm{I}}$ and $\epsilon_{\mathrm{T}}$ are exponents describing the current- and temperature-driven critical behaviors near DMT, respectively, and $b \equiv|f-1|$.

Using the formal procedure introduced in Ref. [21] for determining these critical exponents based on evaluating the 
(a)

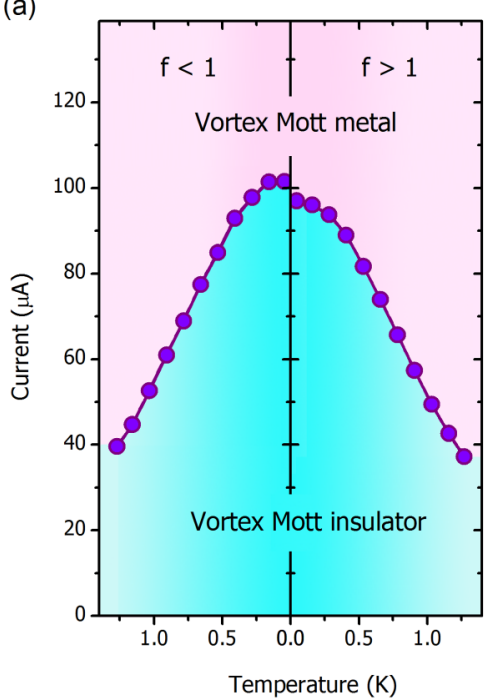

(b)

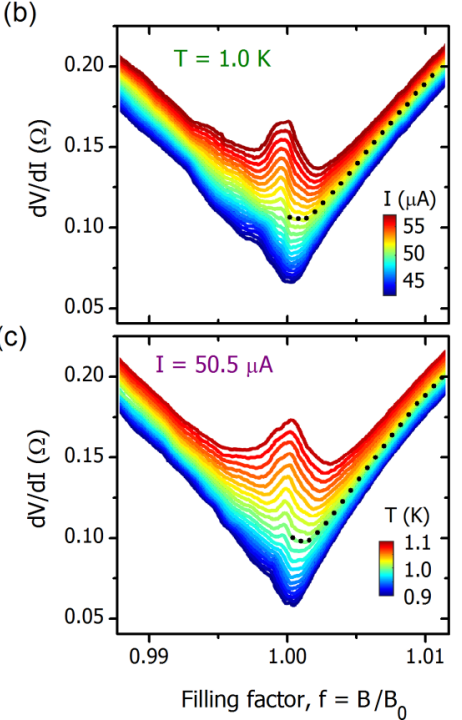

(d)

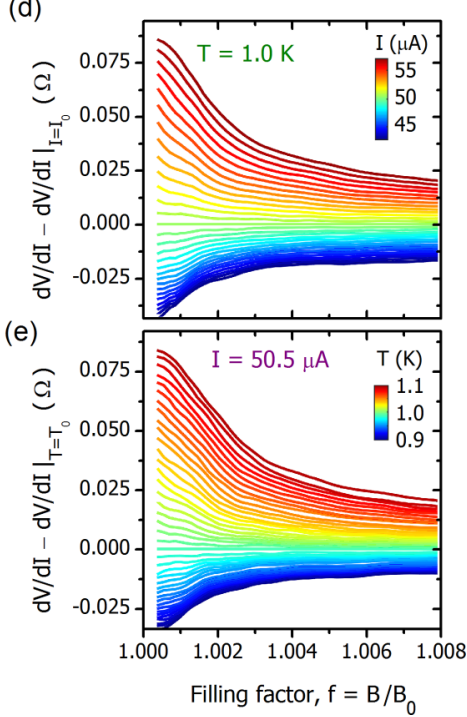

FIG. 2. Vortex dynamic Mott insulator-to-metal transition. (a) The temperature-current phase diagram of the vortex Mott states. The left and right panels present the transition line between the insulating and metallic states at $f<1$ and $f>1$, respectively. In the former, the elementary excitations are vortex holes, i.e., some of the traps lack vortices. In the latter, the elementary excitations are the excess vortices, i.e., some traps contain more than one vortex. (b) A set of differential resistance vs filling factor curves taken at different currents in the critical region at temperature $T=1.0 \mathrm{~K}$. The set corresponds to a currentwise crossing of the phase boundary. The currents increase from the bottom to the top; the range of currents is shown in the color legend. The black dotted line is the separatrix $d V /\left.d I\right|_{I=I_{0}}, I_{0}=51.0 \mu \mathrm{A}$, for $f>1$. The separatrix divides current ranges corresponding to the vortex Mott insulator (at $I<I_{0}, d V / d I$ bends down as $f \rightarrow 1$ ) and vortex Mott metal (at $I>I_{0}, d V / d I$ turning up as $f \rightarrow 1$ ). (c) A similar set of differential resistances vs filling factor curves, but taken at different temperatures and fixed current $I=50.5 \mu \mathrm{A}$. The temperature increases from the bottom to the top and corresponds to the temperaturewise crossing of the phase boundary line. The black dotted line is the separatrix $d V /\left.d I\right|_{T=T_{0}}, T_{0}=1.0 \mathrm{~K}$, for $f>1$. (d), (e) The differential magnetoresistances $d V / d I$ after subtracting the separatrices $d V /\left.d I\right|_{I=I_{0}}$ and $d V /\left.d I\right|_{T=T_{0}}$, respectively. The fanlike sets of curves visualize the dynamic Mott transition.

derivative of the dynamic resistance with respect to $I$ (or $T$ ) at its critical value $I_{0}\left(T_{0}\right)$, we arrive at

$$
[\partial(d V / d I) / \partial I]_{I_{0}} \propto b^{-\epsilon_{\mathrm{I}}}, \quad[\partial(d V / d I) / \partial T]_{T_{0}} \propto b^{-\epsilon_{\mathrm{T}}} .
$$

Plotting $[\partial(d V / d I) / \partial I]_{I_{0}}$ and $[\partial(d V / d I) / \partial T]_{T_{0}}$ as functions of $b$ on a log-log scale should yield straight lines with slopes equal to $-\epsilon_{\mathrm{I}}$ and $-\epsilon_{\mathrm{T}}$, respectively. The results of this procedure are displayed in Figs. 4(a) and 4(b). The data are indeed the straight lines for both current and temperature derivatives, and
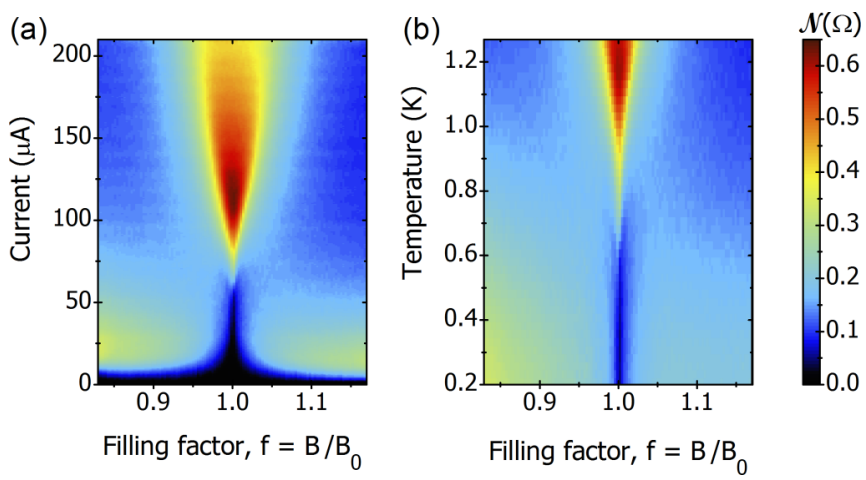

FIG. 3. The critical region of the vortex dynamic Mott transition. The color plots of the measure of degree of nonlinearity $\mathcal{N}=$ $d V / d I-V / I$ as a function of the filling factor $f$ and current at $T=1.0 \mathrm{~K}$ (a) and as function of $f$ and temperature at $I=90 \mu \mathrm{A}$ (b). The color legend is the same for both plots. the linear fit yields exponent values $\epsilon_{\mathrm{I}}=0.64 \pm 0.02$ and $\epsilon_{\mathrm{T}}=$ $0.63 \pm 0.03$. Note that the approach employed for determining the scaling exponents uses only the values of the derivatives of the dynamic resistance at the critical point as given by Eq. (3). This gives us a good starting point for the scaling analysis of the entire set of data following Eqs. (1) and (2). In Figs. 4(c) and 4(d) we plot the data of Figs. 2(b) and 2(c) as functions of the scaling variables $\left|I-I_{0}\right| / b^{\epsilon_{\mathrm{I}}}$ and $\left|T-T_{0}\right| / b^{\epsilon_{\mathrm{T}}}$, respectively. The collapse of the data on the single curves is excellent, over two orders of magnitude of scaled abscissas for identical values of exponents $\epsilon_{\mathrm{I}}=2 / 3$ and $\epsilon_{\mathrm{T}}=2 / 3$. The same $\epsilon_{\mathrm{I}}$ for the current-driven transition was reported previously [14] for a similar proximity system, but with distinctly different parameters. Namely, the critical temperature was significantly higher, $7.3 \mathrm{~K}$, and the island separation was smaller by a factor of 2 compared to the present case. More results of the scaling analysis supporting the universality of the critical exponents are given in SM. As a next step we superimpose the scaling curves from Figs. 4(c) and 4(d) by dividing the temperature abscissa by the factor $r=1.5 \times 10^{4} \mathrm{~K} / \mathrm{A}$, which on a $\log$ scale corresponds to a mere shift of the curves [see Fig. 4(e)]. The striking collapse of the isocurrent and isothermal scaling curves heralds universality of the critical scaling at the DMT. The identity of the scaling functions $\mathcal{F}_{\mathrm{I}}$ and $\mathcal{F}_{\mathrm{T}}$ from Eqs. (1) and (2), together with the equality $\epsilon_{\mathrm{I}}=\epsilon_{\mathrm{T}}$, establishes the interchangeability of the temperature and current effects in the critical region. Finally, the collapse evidences the linear relation between the current- and temperature-induced 
(a)

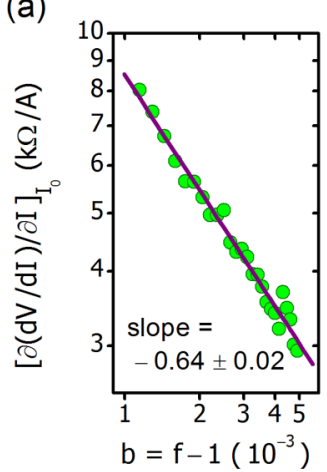

(b)

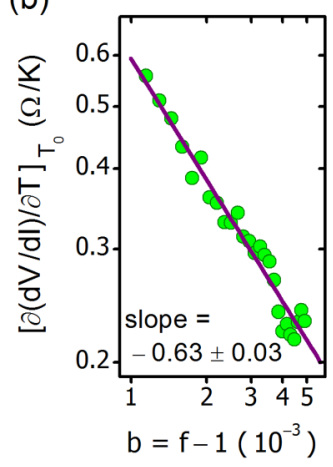

(c)

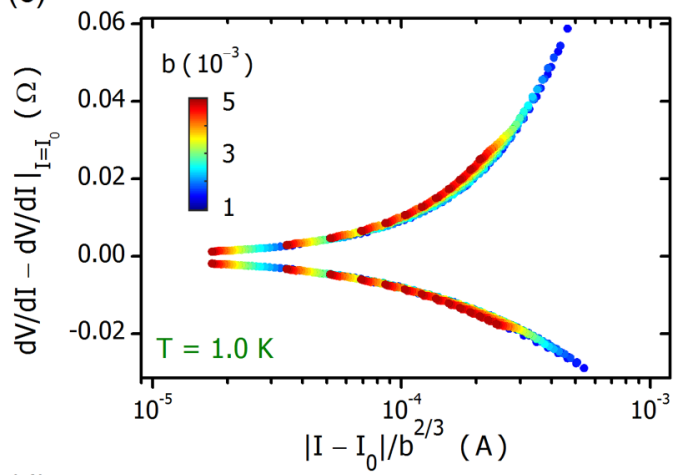

(d)

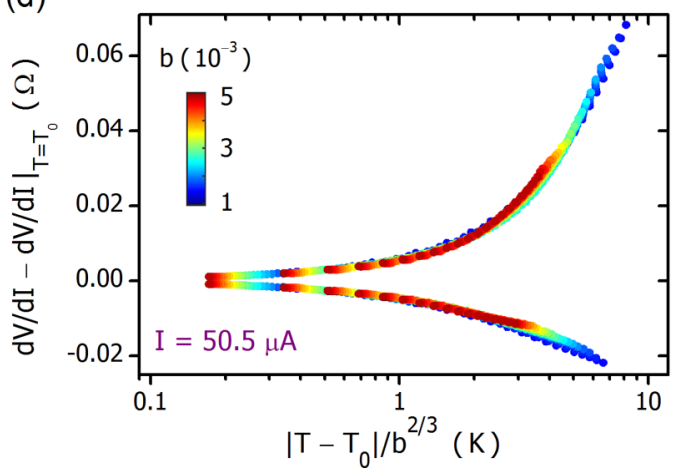

(e)

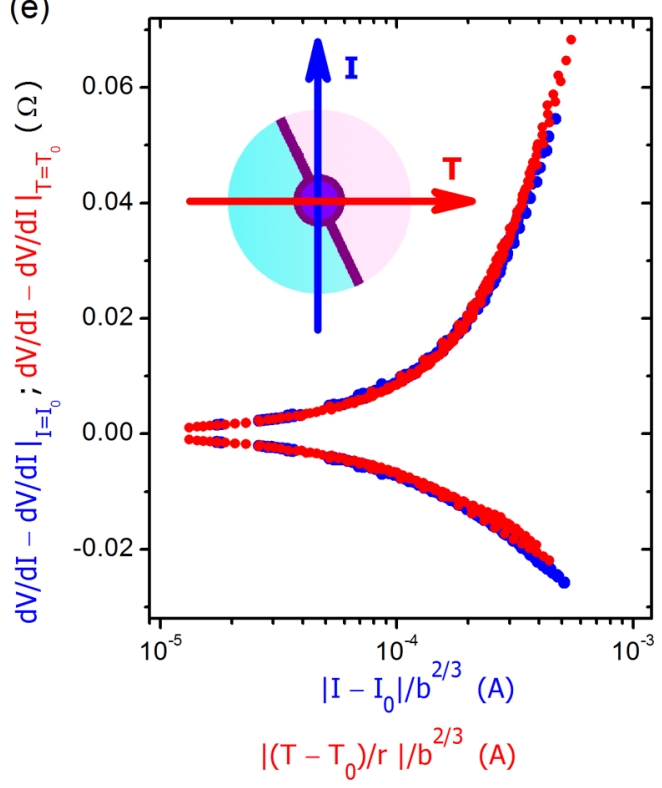

FIG. 4. Scaling analysis of the dynamic Mott transition. (a), (b) The log-log plots of $[\partial(d V / d I) / \partial I]_{I_{0}}$ and of $[\partial(d V / d I) / \partial T]_{T_{0}}$ vs $b$, both shown by symbols. The solid lines show the linear fits. (c) The semilog plot of the differential magnetoresistances $d V / d I$ after subtracting the separatrix $d V /\left.d I\right|_{I=I_{0}}$ presents the same data as Fig. 2(d) as a function of the scaling variable $\left|I-I_{0}\right| / b^{2 / 3}$. The perfect collapse onto two generic scaling curves for $I<I_{0}$ and $I>I_{0}$ at $\epsilon_{\mathrm{I}}=2 / 3$ evidences the critical behavior of the current-driven vortex Mott transition. (d) The semilog plot of the differential magnetoresistances $d V / d I$ after subtracting the separatrix $d V /\left.d I\right|_{T=T_{0}}$ presents the same data as Fig. 2(e) as a function of the scaling variable $\left|T-T_{0}\right| / b^{2 / 3}$. This illustrates the critical behavior of the temperature-driven crossing of the DMT transition line. (e) The plots from (c) (blue symbols) and (d) (red symbols) perfectly collapse on top of each other upon rescaling the abscissa of (d) by a factor $1 / r$ with $r=1.5 \times 10^{4} \mathrm{~K} / \mathrm{A}$, evidencing the identity of the $\mathcal{F}_{\mathrm{I}}$ and $\mathcal{F}_{\mathrm{T}}$ scaling functions defined by Eqs. (1) and (2). The inset shows the segment of the phase transition line. The blue and red arrows stand for current-driven and temperature-driven crossings of the transition line, respectively.

effects and rules out the heating origin of the current-driven transition.

Discussion. The experimental value $\epsilon_{\mathrm{T}}=2 / 3$ coincides with a similar exponent for the thermodynamic Mott transition in an electronic system [22]. This implies that the thermodynamic Mott critical behavior extends onto farfrom-equilibrium DMT. Following the ideas of Ref. [16], we generalize the derivation of the Landau functional in Ref. [18] onto the DMT by including the driving current on the same footing as temperature. In the presence of the current, the linear form eliminating the quadratic term in the Landau functional for the order parameter generalizes to $\mathcal{L}\left(|f-1|, T-T_{0}, I-I_{0}\right) \equiv \operatorname{const}_{f}(f-1)+\operatorname{const}_{T}(T-$ $\left.T_{0}\right)+\operatorname{const}_{I}\left(I-I_{0}\right)$. Accordingly, the condition that $\mathcal{L}=$ 0 near the transition [18] implies that $\operatorname{const}_{T}\left(T-T_{0}\right)+$ const $_{I}\left(I-I_{0}\right)=0$ if we put $f=1$. This gives rise to $\left(T_{0}-\right.$ $T) /\left(I_{0}-I\right)=-$ const $\simeq\left(d T_{0} / d I_{0}\right)$. Making use of the phase diagram in Fig. 2(a), one finds, at $T_{0}=1.0 \mathrm{~K},\left(d T_{0} / d I_{0}\right)=$ $1.7 \times 10^{4} \mathrm{~K} / \mathrm{A}$, in fair agreement with the experimental rescaling factor $1.5 \times 10^{4} \mathrm{~K} / \mathrm{A}$. To understand the meaning of this factor, we recall that the energy that sets the depth of the potential well localizing vortices is estimated for a square sinusoidal egg-crate potential as $0.2 E_{J}$ [23], where the Josephson coupling of a single junction $E_{J}=(\hbar / 2 e) i_{c}$, $i_{c}=I_{c} /(N-1)$ is the critical current for a single junction, $I_{c}$ is the critical current of the array, and $N$ is the number of rows in the array. In this case we find that the fundamental temperature-to-current conversion ratio for a Josephson junction array $[T / I] \equiv 0.2 E_{J} /\left[(N-1) k_{\mathrm{B}} i_{c}\right]=1.77 \times$ $10^{4} \mathrm{~K} / \mathrm{A}$, which nicely compares with the experimental $r=$ $1.5 \times 10^{4} \mathrm{~K} / \mathrm{A}$. That $[T / I] \gtrsim r$ indicates that the dielectric breakdown of the Mott insulator occurs under the condition that vortices are still pinned, which is in accord with our direct observation.

Identical scaling functions and the resulting interchangeability of current and temperature have far-reaching consequences, most notably, that the dynamic critical behavior of the Mott transition would teach us about thermodynamic criticality as well. To describe the scaling, we, following the approach of Ref. [18], write the Ginzburg-Landau free-energy functional density for the fluctuating part of the vortex system near $f=1$ as (see SM)

$$
\mathcal{F}=D|\nabla \Psi|^{2}+m^{2}|\Psi|^{2}+u|\Psi|^{4}
$$

where $D$ is the stiffness of the excess vortices system, and $m$ and $u$ are respectively the mass and interaction parameters 
that govern the mean-field transition. Then, the equation for overdamped vortex motion assumes the form

$$
\frac{\partial \Psi}{\partial t}-i(I / \rho) x \Psi=D \nabla^{2} \Psi-m^{2} \Psi-2 u|\Psi|^{2} \Psi
$$

where $\rho$ represents viscous damping, and $I$ is the applied imaginary current driving vortices that appeared as a result of the gauge transformation transforming the vector potential into a scalar one. This equation is formally identical to a nonlinear Schrödinger equation in Euclidean time for a particle subject to an imaginary electric field. Applying the technique of Refs. [24,25] (see also Ref. [20]), we find $\left(I_{0}-I\right)^{3 / 2}$ scaling near the DMT, in full accord with experimental observations. Note that our description of the DMT rests on the general properties of non-Hermitian Hamiltonians rather than on the peculiarities of Mott systems. A general principle is that if at small drives the open dissipative system's non-Hermitian Hamiltonian is endowed with $\mathcal{P} \mathcal{T}$ symmetry, the out-of-equilibrium phase transition manifests as a $\mathcal{P} \mathcal{T}$ symmetry breaking of the eigenstates at some threshold driving field.

We thank Frank Roesthuis and Dick Veldhuis for help and support during the experiments, and I. Aleiner, B. Altshuler, G. Kotliar, and A. Millis for illuminating discussions. Work was supported by the Dutch FOM and NWO foundations, the Italian Ministry for Education and Research, the Russian Science Foundation (Project No. 14-22-00143), the Ministry of Education and Science of the Russian Federation, Grant No. 14.Y26.31.0007, and by the U.S. Department of Energy, Office of Science, Materials Sciences and Engineering Division (A.G. and V.M.V.); V.T. was supported through Materials Theory Institute at ANL, the University of Chicago Center in Delhi, and a DST (India) Swarnajayanti grant (No. DST/SJF/PSA0212012-13). T.I.B. acknowledges financial support from the Alexander von Humboldt Foundation and from the Consejería de Educación, Cultura y Deporte (Comunidad de Madrid) through the talent attraction program, Ref. No. 2016-T3/IND1839.
[1] N. F. Mott and R. Peierls, Discussion of the paper by de Boer and Verwey, Proc. Phys. Soc. 49, 72 (1937).

[2] N. F. Mott, The basis of the electron theory of metals with special reference to the transition metals, Proc. Phys. Soc. A 62, 416 (1949).

[3] N. F. Mott, Metal-Insulator Transitions (Taylor and Francis, London, 1990).

[4] S. Sachdev, Quantum Phase Transitions, 2nd ed. (Cambridge University Press, Cambridge, UK, 2011).

[5] M. Imada, A. Fujimori, and Y. Tokura, Metal-insulator transitions, Rev. Mod. Phys. 70, 1039 (1998).

[6] Conductor-Insulator Quantum Phase Transitions, edited by V. Dobrosavljevic, N. Trivedi, and J. M. Valles, Jr. (Oxford University Press, Oxford, UK, 2012).

[7] L. Balents, L. Bartosch, A. Burkov, S. Sachdev, and K. Sengupta, Competing Orders and Non-Landau-Ginzburg-Wilson Criticality in (Bose) Mott Transitions, Prog. Theor. Phys. Suppl. 160, 314 (2005).

[8] P. A. Lee, N. Nagaosa, and X.-G. Wen, Doping a Mott insulator: Physics of high temperature superconductivity, Rev. Mod. Phys. 78, 17 (2006).

[9] A. M. Polyakov, Gauge Fields and Strings (Harwood Academic, Chur, 1987).

[10] D. R. Nelson and V. M. Vinokur, Boson localization and correlated pinning of superconducting vortex arrays, Phys. Rev. B 48, 13060 (1993).

[11] R. A. Lehrer and D. R. Nelson, Vortex pinning and the non-Hermitian Mott transition, Phys. Rev. B 58, 12385 (1998).

[12] M. Baert, V. V. Metlushko, R. Jonckheere, V. V. Moshchalkov, and Y. Bruynseraede, Composite Flux-Line Lattices Stabilized in Superconducting Films by a Regular Array of Artificial Defects, Phys. Rev. Lett. 74, 3269 (1995).

[13] S. Goldberg, Y. Segev, Y. Myasoedov, I. Gutman, N. Avraham, M. Rappaport, E. Zeldov, T. Tamegai, C. W. Hicks, and K. A. Moler, Mott insulator phases and first-order melting in
$\mathrm{Bi}_{2} \mathrm{Sr}_{2} \mathrm{CaCu}_{2} \mathrm{O}_{8+\delta}$ crystals with periodic surface holes, Phys. Rev. B 79, 064523 (2009).

[14] N. Poccia, T. I. Baturina, F. Coneri, C. G. Molenaar, X. R. Wang, G. Bianconi, A. Brinkman, H. Hilgenkamp, A. A. Golubov, and V. M. Vinokur, Critical behavior at a dynamic vortex insulatorto-metal transition, Science 349, 1202 (2015).

[15] A. Mitra, S. Takei, Y.-B. Kim, and A. J. Millis, Nonequilibrium Quantum Criticality in Open Electronic Systems, Phys. Rev. Lett. 97, 236808 (2006).

[16] N. M. Chtchelkatchev and V. M. Vinokur, Nonequilibrium mesoscopic superconductors in a fluctuational regime, Europhys. Lett. 88, 407 (2009).

[17] J. Hubbard, Electron correlations in narrow energy bands, Proc. R. Soc. London, Ser. A 276, 238 (1963).

[18] G. Kotliar, E. Lange, and M. J. Rozenberg, Landau Theory of the Finite Temperature Mott Transition, Phys. Rev. Lett. 84, 5180 (2000).

[19] M. J. Rozenberg, R. Chitra, and G. Kotliar, Finite Temperature Mott Transition in the Hubbard Model in Infinite Dimensions, Phys. Rev. Lett. 83, 3498 (1999).

[20] See Supplemental Material at http://link.aps.org/supplemental/ 10.1103/PhysRevB.97.020504 for details of the measurements and the technical derivation of the theoretical results, which includes Refs. [16,26-34].

[21] A. F. Hebard and M. A. Paalanen, Magnetic-field-tuned superconductor-insulator transition in two-dimensional films, Phys. Rev. Lett. 65, 927 (1990).

[22] P. Limelette, A. Georges, D. Jérome, P. Wzietek, P. Metcalf, and J. M. Honig, Universality and Critical Behavior at the Mott Transition, Science 302, 89 (2003).

[23] C. J. Lobb, D. W. Abraham, and M. Tinkham, Theoretical interpretation of resistive transition data from arrays of superconducting weak links, Phys. Rev. B 27, 150 (1983).

[24] N. M. Chtchelkatchev, A. A. Golubov, T. I. Baturina, and V. M. Vinokur, Stimulation of the Fluctuation Superconductivity by PT Symmetry, Phys. Rev. Lett. 109, 150405 (2012). 
[25] V. Tripathi, A. Galda, H. Barman, and V. M. Vinokur, Parity-time symmetry-breaking mechanism of dynamic Mott transitions in dissipative systems, Phys. Rev. B 94, 041104(R) (2016).

[26] A. M. Dykhne, Adiabatic perturbation of discrete spectrum states, JETP 14, 941 (1962).

[27] T. Oka and H. Aoki, Dielectric breakdown in a Mott insulator: Many-body Schwinger-Landau-Zener mechanism studied with a generalized Bethe ansatz, Phys. Rev. B 81, 033103 (2010).

[28] F. B. Müller-Allinger and A. C. Mota, Reentrance of the induced diamagnetism in gold-niobium proximity cylinders, Phys. Rev. B 62, R6120(R) (2000).

[29] V. M. Vinokur, B. Khaykovich, E. Zeldov, M. Konczykowski, R. A. Doyle, and P. H. Kes, Lindemann criterion and vortex-matter phase transitions in high-temperature superconductors, Physica C 295, 209 (1998).
[30] G. Blatter, M. V. Feigel'man, V. B. Geshkenbein, A. I. Larkin, and V. M. Vinokur, Vortices in high-temperature superconductors, Rev. Mod. Phys. 66, 1125 (1994).

[31] T. Oka, R. Arita, and H. Aoki, Breakdown of a Mott Insulator: A Nonadiabatic Tunneling Mechanism, Phys. Rev. Lett. 91, 066406 (2003).

[32] M. Eckstein, T. Oka, and P. Werner, Dielectric Breakdown of Mott Insulators in Dynamical Mean-Field Theory, Phys. Rev. Lett. 105, 146404 (2010).

[33] J. Rubinstein, P. Sternberg, and Q. Ma, Bifurcation Diagram and Pattern Formation of Phase Slip Centers in Superconducting Wires Driven with Electric Currents, Phys. Rev. Lett. 99, 167003 (2007).

[34] C. M. Bender and S. Boettcher, Real Spectra in Non-Hermitian Hamiltonians Having PT Symmetry, Phys. Rev. Lett. 80, 5243 (1998). 